\title{
CONVERGENCE GROUPS, HAUSDORFF DIMENSION, AND A THEOREM OF SULLIVAN AND TUKIA
}

\author{
JAMES W. ANDERSON, PETRA BONFERT-TAYLOR, AND EDWARD C. TAYLOR
}

\begin{abstract}
We show that a discrete, quasiconformal group preserving $\mathbb{H}^{n}$ has the property that its exponent of convergence and the Hausdorff dimension of its limit set detect the existence of a non-empty regular set on the sphere at infinity to $\mathbb{H}^{n}$. This generalizes a result due separately to Sullivan and Tukia, in which it is further assumed that the group act isometrically on $\mathbb{H}^{n}$, i.e. is a Kleinian group. From this generalization we are able to extract geometric information about infinite-index subgroups within certain of these groups.
\end{abstract}

\section{Introduction and Statement of Results}

The purpose of this note is to probe whether certain well-known results in Patterson-Sullivan theory depend on the assumption that the discrete group in question has an isometric action. In this study we consider the class of discrete $K$ quasiconformal groups acting on $\overline{\mathbb{R}^{n}}$ that preserve hyperbolic $n$-space $\mathbb{H}^{n}$; if $K>1$, these groups do not act isometrically on $\mathbb{H}^{n}$. Patterson-Sullivan theory itself is revealing of the deep relationship between the conformal and isometric action of a Kleinian group. In particular, various geometric and conformal invariants (e.g. the exponent of convergence of the Poincaré series, the Hausdorff dimension of the limit set, the lowest eigenvalue of the Laplacian) are intertwined by this theory to give a full description of both the dynamical and geometric properties of a large class of Kleinian groups.

Recall that a Kleinian group is a discrete group of orientation-preserving isometries of hyperbolic $n$-space; we use the upper half-space model $\left(\mathbb{H}^{n}, \rho\right)$ of hyperbolic space, where $\mathbb{H}^{n}=\left\{\left(x_{1}, \ldots, x_{n}\right) \in \mathbb{R}^{n}: x_{n}>0\right\}$ and where the distance $\rho$ is induced by the metric $\mathrm{ds}^{2}=\frac{1}{x_{n}^{2}}\left(\mathrm{dx}_{1}^{2}+\cdots+\mathrm{dx}_{n}^{2}\right)$. The isometric action of a Kleinian group on $\mathbb{H}^{n}$ extends to a conformal action on the sphere at infinity $\overline{\mathbb{R}^{n-1}} \simeq\left(\mathbb{R}^{n-1} \times\{0\}\right) \cup\{\infty\}$. This conformal action partitions $\overline{\mathbb{R}^{n-1}}$ into two disjoint sets; the regular set $\Omega(\Gamma)$ is the largest open set on which $\Gamma$ acts discontinuously, and the limit set $L(\Gamma)$ is its complement. The Hausdorff dimension of the limit set of a Kleinian group is descriptive of the geometric action of the group, e.g. see [3], [11], and [12]. Most basically, if $\Gamma$ is geometrically finite, then the Hausdorff dimension of the limit set detects whether $\Gamma$ has a discontinuous action on some subset of $\overline{\mathbb{R}^{n-1}}$. (For the basics in Patterson-Sullivan theory as applied to Kleinian groups see Nicholls [20].)

Theorem 1.1. (Sullivan [23] and Tukia [25]) Let $\Gamma$ be a geometrically finite Kleinian group acting on $\mathbb{H}^{n}$. Then $\operatorname{dim} L(\Gamma)=n-1$ if and only if $L(\Gamma)=\overline{\mathbb{R}^{n-1}}$.

Date: May 18, 2006. 
If $\Gamma$ is a geometrically finite Kleinian group, then the Hausdorff dimension of its limit set is equal to the exponent of convergence of its Poincaré series; thus this theorem of Sullivan and Tukia could have equivalently been phrased in terms of the exponent of convergence.

Theorem 1.1, as stated with respect to both Hausdorff dimension and the exponent of convergence, is the theorem we will be generalizing to a class of discrete nonisometric actions on $\mathbb{H}^{n}$. Recall that a discrete $K$-quasiconformal group $G$ acting on $\overline{\mathbb{R}^{n}}$ is a discrete group of homeomorphisms, each of which is a $K$-quasiconformal mapping. We note that a discrete 1-quasiconformal group is in fact a discrete group of Möbius transformations, i.e. a Kleinian group. Because we frequently wish to use geometric arguments involving the hyperbolic metric $\rho$, we often consider those discrete $K$-quasiconformal groups acting on $\overline{\mathbb{R}^{n}}$ that preserve the upper half-space $\mathbb{H}^{n}$. We call such groups quasiconformal Fuchsian groups. (We denote such groups with the initials "QCF".) Quasiconformal groups, and indeed the more general class of convergence groups, share many of the basic properties of Kleinian groups, e.g. the notions of limit set and regular set, the dynamical classification of group elements, etc. Most importantly, there is a dynamical notion of a geometrically finite action. See the following section for relevant definitions and facts concerning the analytic and geometric properties of convergence groups.

As in the Kleinian group setting, there is a well-defined notion of an exponent of convergence $\delta(G)$ of the Poincaré series of a QCF group $G([6])$. However, the sharpest statement one can make concerning its relationship to the limit set for QCF groups is that the exponent of convergence bounds from above the Hausdorff dimension of the limit set (see [6]).

We are now prepared to state the central results of this note.

Theorem 1.2. Let $G$ be a discrete quasiconformal Fuchsian group acting on $\overline{\mathbb{R}^{n}}$ having non-empty regular set in $\overline{\mathbb{R}^{n-1}}$ and having a purely conical limit set. Then $\delta(G)<n-1$.

Having a purely conical limit set is a dynamical restriction on the action of the QCF group; see section 2. We remark that if $G$ is a non-elementary QCF group, then $\delta(G)>0$ (see either Gehring-Martin [16] or [6]).

One could use Theorem 1.2 and the results from [6] to show that $\operatorname{dim} L(G)<$ $n-1$ for discrete QCF groups satisfying the hypotheses of Theorem 1.2. However, it is possible to prove this bound on the Hausdorff dimension of the limit set directly for a more general class of discrete quasiconformal groups, that is, for those that do not necessarily preserve $\mathbb{H}^{n}$.

Theorem 1.3. Let $G$ be a discrete quasiconformal group acting on $\overline{\mathbb{R}^{n}}$ having nonempty regular set and having a purely conical limit set. Then $\operatorname{dim} L(G)<n$.

As an application of these theorems, we are able to show how infinite-index subgroups reside measure theoretically within certain classes of discrete quasiconformal groups, see Section 4.

Acknowledgements: We thank Chris Bishop for the argument showing Example 4.6. The second author is partially funded via NSF grant 0070335. The first and third authors were partially funded by grants from EPSRC. 


\section{BASICS}

Recall that a discrete convergence group $G$ acting on a compact perfect metric space $M$ is a discrete (with respect to the compact-open topology) group of homeomorphisms acting on $M$ that has the following convergence property: For every sequence $\left\{g_{j}\right\}$ in $G$, there is a subsequence $\left\{g_{j_{k}}\right\}$ and two (not necessarily distinct) points $a, b \in M$ such that $g_{j_{k}}(x) \rightarrow a$ and $g_{j_{k}}^{-1}(y) \rightarrow b$ locally uniformly in $M-\{b\}$ and $M-\{a\}$ respectively. The notion of a convergence group was developed by Gehring and Martin ([15]); in that paper the authors began a program in which they showed that these groups resemble their conformal counterparts in many essential ways. One defines the limit set $L(G)$, the regular set $\Omega(G)$ and the notion of being non-elementary exactly as in the Kleinian case ([18]). Note that, for a non-elementary discrete convergence group, the limit set is perfect and closed, as in the Kleinian case. Gehring and Martin also show that a discrete $K$-quasiconformal group is necessarily a convergence group ([15]), and thus a discrete non-elementary quasiconformal group acts as a convergence group on its limit set.

The analogue of geometric finiteness that is most appropriate for this paper is dynamical in nature. Recall that a conical limit point of a discrete convergence group $G$ acting on $M$ is a point $x \in M$ for which there exists a sequence $\left\{g_{k}\right\}$ in $G$ and distinct points $a, b \in M$ so that $g_{k}(x) \rightarrow a$ but $g_{k}(y) \rightarrow b$ for all $y \in M-\{x\}$. In the case of a Kleinian group acting on $\overline{\mathbb{R}^{n-1}}$ and naturally extending to hyperbolic space $\mathbb{H}^{n}$, this definition is equivalent to the existence of a sequence $\left\{g_{k}\right\}$ in $G$ such that $\left\{g_{k}(j)\right\}$ converges to $x$ within a bounded hyperbolic distance of the infinite directed hyperbolic ray from $j$ in the direction of $x$. A Kleinian group with purely conical limit set is geometrically finite, that is to say that any epsilon neighborhood of its convex core has finite (hyperbolic) volume. For a complete discussion of geometrical finiteness as applied to Kleinian groups see Bowditch [9].

As mentioned previously, we will occasionally wish to use geometric arguments involving the hyperbolic metric; as such, we will sometimes restrict our consideration to QCF groups. We identify $\partial \mathbb{H}^{n}$ with $\overline{\mathbb{R}^{n-1}}$; if $G$ is a quasiconformal group preserving $\mathbb{H}^{n}$ then $\partial \mathbb{H}^{n}$ is also invariant under $G$. If one furthermore assumes that $G$ is discrete then the action of $G$ is discontinuous in $\mathbb{H}^{n}$ and $L(G) \subseteq \overline{\mathbb{R}^{n-1}}$ by Corollary 3.8 in [15]. As for Kleinian groups we define the Poincaré series of a discrete QCF group $G$ to be

$$
\sum_{g \in G} e^{-s \rho(j, g(j))}
$$

where $j=(0, \ldots, 1) \in \mathbb{H}^{n}$. The exponent of convergence $\delta(G)$ is

$$
\delta(G)=\inf \left\{s \geq 0: \sum_{g \in G} e^{-s \rho(j, g(j))}<\infty\right\} ;
$$

the exponent of convergence does not depend on the choice of base point $j \in \mathbb{H}^{n}$ (see $[6])$.

In the geometric study of Kleinian groups, the fact that the conformal action extends to a properly discontinuous and isometric action on $\mathbb{H}^{n}$ is foundational. No such nice property exists for convergence groups in general, however there is a space that serves as a partial substitute for $\mathbb{H}^{n}$. One can replace "hyperbolic space" by the triple space of the underlying metric space: A discrete convergence group $G$ acting on a compact perfect metric space $M$ induces a properly discontinuous action on the triple space $T(M)=\{(x, y, z) \mid x, y, z \in M$ distinct $\}$ by the diagonal action of $G$ 
on $T(M)$ given by $g((x, y, z)):=(g(x), g(y), g(z))$. Here, $T(M)$ inherits the product topology from $M \times M \times M$, and can be naturally compactified by adjoining a copy of $M$. This can be described by regarding $T(M) \cup M$ as a quotient of $M \times M \times M$, where the quotient map is the identity on $T(M)$, and sends a triple $(x, y, z)$ to $a \in M$ if at least two of the entries $x, y, z$ are equal to $a$. Then a sequence $\left\{X_{i}\right\}$ in $T(M)$ converges (in $T(M) \cup M$ ) to $a \in M$ if and only if two of its components converge (in $M$ ) to $a$. For a detailed discussion of the triple space of a convergence group, see Bowditch [10] and Tukia [26].

The central fact we will use concerning the triple space is that the dynamical action of a convergence group can be reflected in the topological action of the group on a triple space.

Theorem 2.1. (Bowditch [10] and Tukia [26]) Let $G$ be a discrete convergence group acting on a perfect compact metric space $M$. Then $T(M) / G$ is compact if and only if every point of $M$ is a conical limit point.

\section{Proofs of Theorems 1.2 And 1.3}

We begin with the proof of Theorem 1.3. A certain fact which is shown within this proof (see Corollary 3.2) will later be used in the proof of Theorem 1.2.

Let a $n$-dimensional cube of arbitrary side length be called a $n$-cube. Recall that a bounded set $A \subset \mathbb{R}^{n}$ is porous if there exists a constant $c>0$ such that for any open ball $B(a, r)$ centered at a point $a \in A$ and of radius $r \in(0,1]$, there is an open ball $B(b, c r)$ contained in $B(a, r)$ and disjoint from $A$. It follows from a standard argument (see for example [19]) that a porous subset of $\mathbb{R}^{n}$ has Hausdorff dimension strictly less than $n$.

To prove Theorem 1.3 we adapt an argument of Tukia [25] to the triple space of a discrete quasiconformal group $G$.

Proof of Theorem 1.3. If $G$ is elementary then the result is trivially established; thus we will assume for the rest of the argument that $G$ is non-elementary. In particular we then know that $L(G)$ is a compact perfect subset of $\overline{\mathbb{R}^{n}}$. Since $\Omega(G) \neq \emptyset$ we can assume, by conjugating with a Möbius transformation, that $\infty \in \Omega(G)$ so that we now have that $L(G)$ is a perfect, closed and bounded subset of $\mathbb{R}^{n}$.

Let $K$ be a bound on the quasiconformal distortion of the elements of $G$ (i.e. $G$ is a $K$-quasiconformal group). We will show: There exists an integer $q$ (the so-called porosity index) such that if we divide any $n$-cube $Q$ in $\mathbb{R}^{n}$ into $q^{n}$ sub-cubes of equal side length then at least one of these sub-cubes does not intersect $L(G)$. That is, we will show that $L(G)$ is porous, and thus it follows that $\operatorname{dim} L(G)<n$.

To show the existence of the integer $q$, let $T_{G}$ denote the triple space over $L(G)$, i.e. $T_{G}=T(L(G))=\left\{\left(x_{1}, x_{2}, x_{3}\right) \mid x_{1}, x_{2}, x_{3} \in L(G)\right.$ distinct $\}$. Since $L(G)$ is purely conical, we know from Theorem 2.1 that $T_{G} / G$ is compact, and so there exists a compact subset $C \subset T_{G}$ with $G(C)=T_{G}$. Since $C$ is compact in $T_{G}$ there exists an $\varepsilon>0$ such that if $\left(x_{1}, x_{2}, x_{3}\right) \in C$ then $\left|x_{i}-x_{j}\right| \geq \varepsilon$ for $i \neq j$, i.e. the coordinates of a point in $C$ are uniformly separated from each other.

Choose a standard (fixed) $n$-cube $Q_{0}$ of unit side length in $\mathbb{R}^{n}$.

Let $\mathcal{Q}$ be the collection of all cubes in $\mathbb{R}^{n}$ for which $q=4$ does not work, i.e. if $Q \in \mathcal{Q}$ and $Q$ is divided into $4^{n}$ subcubes of equal side length, then each sub-cube intersects $L(G)$. 
Fix $Q \in \mathcal{Q}$, then there are three points $x_{1}^{(Q)}, x_{2}^{(Q)}, x_{3}^{(Q)} \in L(G) \cap Q$ such that the distances $\left|x_{i}^{(Q)}-x_{j}^{(Q)}\right|,(i \neq j)$ are all at least $\frac{\text { sidelength }(Q)}{2}$. In particular, the triple $\left(x_{1}^{(Q)}, x_{2}^{(Q)}, x_{3}^{(Q)}\right)$ belongs to the triple space $T_{G}$, and so there exists $g_{Q} \in G$ such that $g_{Q}\left(\left(x_{1}^{(Q)}, x_{2}^{(Q)}, x_{3}^{(Q)}\right)\right)=\left(g_{Q}\left(x_{1}^{(Q)}\right), g_{Q}\left(x_{2}^{(Q)}\right), g_{Q}\left(x_{3}^{(Q)}\right)\right) \in C$. Let $\beta_{Q}: \mathbb{R}^{n} \rightarrow$ $\mathbb{R}^{n}$ be a similarity with $\beta_{Q}(Q)=Q_{0}$ and $\beta_{Q}(\infty)=\infty$, and define $h_{Q}:=\beta_{Q} \circ g_{Q}^{-1}$ : $\overline{\mathbb{R}^{n}} \rightarrow \overline{\mathbb{R}^{n}}$. Then $h_{Q}$ is a $K$-quasiconformal mapping satisfying

$$
h_{Q}\left(g_{Q}\left(x_{i}^{(Q)}\right)\right)=\beta_{Q}\left(x_{i}^{(Q)}\right), \quad i=1,2,3 .
$$

Since $\beta_{Q}$ is a similarity mapping from $Q$ onto $Q_{0}$, we have that the points $\beta_{Q}\left(x_{i}^{(Q)}\right)$ are a distance of at least $1 / 2$ from each other, and furthermore, by compactness of $C$ in $T_{G}$, the points $g_{Q}\left(x_{i}^{(Q)}\right)$ (which are the coordinates of the point $g_{Q}\left(x_{1}^{(Q)}, x_{2}^{(Q)}, x_{3}^{(Q)}\right) \in C$ ) have distance at least $\varepsilon$ from each other. Furthermore $g_{Q}\left(x_{i}^{(Q)}\right) \in L(G)$, where (by the initial conjugation) $L(G)$ is a compact subset of $\mathbb{R}^{n}$.

We have shown that for each $Q \in \mathcal{Q}$ there exists a K-quasiconformal mapping $h_{Q}$ and three points in a fixed bounded subset of $\mathbb{R}^{n}$ that are uniformly (independently of $Q$ ) separated from each other, and these three points are mapped under $h_{Q}$ onto three points in $Q_{0}$ that again are uniformly (independently of $Q$ ) separated from each other. This implies that the family $\mathcal{F}:=\left\{h_{Q} \mid Q \in \mathcal{Q}\right\}$ is equicontinuous ([27, Thm. 19.4]), and thus normal ([27, Thm. 20.5]). Observe also that limit functions of locally uniformly convergent sequences in $\mathcal{F}$ are non-constant $K$-quasiconformal homeomorphisms.

We will show, by contradiction, that there exists a porosity index $q \in \mathbb{N}(q>4)$ that works for all $Q \in \mathcal{Q}$; thus this value for the index will, by the definition of $\mathcal{Q}$, work for all $n$-cubes in $\mathbb{R}^{n}$. Suppose now that no integer $q$ exists that works for all cubes $Q \in \mathcal{Q}$. Then there are $h_{m} \in \mathcal{F}$ such that $Q_{0} \backslash\left(Q_{0} \cap h_{m}(L(G))\right)$ does not contain a cube of side length $1 / \mathrm{m}$. After passing to a subsequence we may assume that $\left\{h_{m}\right\}$ converges to a non-constant, $K$-quasiconformal limit function $h$. Then $Q_{0} \backslash\left(Q_{0} \cap h(L(G))\right)$ does not contain any cube of arbitrarily small side length, i.e. $Q_{0} \cap h(L(G))$ is dense in $Q_{0}$. But then $h^{-1}\left(Q_{0}\right) \cap L(G)$ is dense in $h^{-1}\left(Q_{0}\right)$; this is a contradiction since $L(G)$ is nowhere dense in $\mathbb{R}^{n}$ by [15, Thm. 4.9]. Hence there exists a uniform porosity index $q$ for all cubes, and thus the Hausdorff dimension of $L(G)$ is strictly less than $n$.

Remark 3.1. The assumption that $G$ is a discrete quasiconformal group allows us to use the necessary normal family argument in the above proof. The result is not true in the more general setting of discrete convergence groups. Consider a homeomorphism $g: S^{1} \rightarrow C \subset \mathbb{R}^{2}$, where $C$ is a closed Jordan curve of Hausdorff dimension 2 . For example, $g$ can be built from the function $f:[0,1] \rightarrow \mathbb{R}^{2}$ satisfying the following three properties.

(1) The endpoints of the interval are fixed by $f$, i.e. $f(0)=0$ and $f(1)=1$.

(2) For each $n \in \mathbb{N}: n \geq 2$ the function $f$ maps the interval $\left[1-\frac{1}{n}, 1-\frac{1}{n+1}\right]$ to a snowflake curve $C_{n}$ so that the endpoints are fixed, i.e. $f\left(1-\frac{1}{n}\right)=1-\frac{1}{n}$ and $f\left(1-\frac{1}{n+1}\right)=1-\frac{1}{n+1}$.

(3) For each such $C_{n}$, we have that $\operatorname{dim} C_{n} \geq 2-\frac{1}{n}$. 
By identifying the endpoints we obtain the desired function $g$ from the function $f$. Using the Schoenflies Theorem the function $g$ extends to a homeomorphism of $\overline{\mathbb{R}^{2}}$. By construction, the Hausdorff dimension of $g\left(S^{1}\right)$ is two.

Let $\Gamma$ be a finitely generated Fuchsian group acting on $\overline{\mathbb{R}^{2}}$, having purely conical limit set and so that $L(\Gamma)=S^{1}$. Let $G$ be the convergence group $G=g \circ \Gamma \circ g^{-1}$; note that $G$ is a purely conical convergence group, has non-empty regular set, and its limit set is $g(L(\Gamma))=g\left(S^{1}\right)$ and thus has dimension 2 .

We isolate an artifact of the proof of Theorem 1.3 that will be useful to us in studying the corresponding fact about the exponent of convergence.

Corollary 3.2. Let $G$ be a discrete quasiconformal group acting on $\overline{\mathbb{R}^{n}}$ with nonempty regular set so that $\infty \in \Omega(G)$, and suppose that $G$ has purely conical limit set. Then the limit set of $G$ is porous.

Because the exponent of convergence does not necessarily equal the Hausdorff dimension of the conical limit set (see Theorem 2.7 and Example 4.1 in [6]), we cannot conclude that the proof of Theorem 1.2 follows trivially from the above theorem.

Preliminary results for the proof of Theorem 1.2 are now assembled. Recall that a quasiconformal mapping $g: \mathbb{H}^{n} \rightarrow \mathbb{H}^{n}$ can only distort the hyperbolic metric by a controlled amount.

Theorem 3.3 (Gehring-Martin [16]). For each $n \in \mathbb{N}, n \geq 2$, and all $K \geq 1$, there exists a homeomorphism $\Phi_{n, K}:[0, \infty) \rightarrow[0, \infty)$ with $\Phi_{n, K}(0)=0$, so that $\Phi_{n, k}$ depends only on $n$ and $K$, and such that any $K$-quasiconformal mapping $g: \mathbb{H}^{n} \rightarrow \mathbb{H}^{n}$ satisfies

$$
\rho(g(z), g(w)) \leq \Phi_{n, K}(\rho(z, w))
$$

for all $z, w \in \mathbb{H}^{n}$.

We show below, for use in a counting argument, that discrete quasiconformal groups exhibit a point-wise lower bound on the injectivity radius with respect to the hyperbolic metric. Recall that $j=(0, \ldots, 1) \in \mathbb{H}^{n}$.

Proposition 3.4. Let $G$ be a discrete quasiconformal group preserving $\mathbb{H}^{n}$. Suppose that $j$ is not fixed by any element of $G$. Then there exists a constant $d>0$ such that the orbit of $j$ under $G$ is uniformly separated by $d$ in the hyperbolic metric, i.e.

$$
\rho(g(j), h(j)) \geq d
$$

whenever $g$ and $h$ are two distinct elements in $G$.

Proof of Proposition 3.4. Suppose that the claim is not true. Then we can find distinct $g_{i}$ and $h_{i}$ in $G$ such that $\rho\left(g_{i}(j), h_{i}(j)\right) \rightarrow 0$ as $i \rightarrow \infty$. By assumption there exists a $K \geq 1$ such that each $g \in G$ is $K$-quasiconformal. Using Theorem 3.3 we then obtain that $\rho\left(j, g_{i}^{-1}\left(h_{i}(j)\right)\right) \rightarrow 0$ as $i \rightarrow \infty$, and this contradicts the fact that $G$ acts discontinuously on $\mathbb{H}^{n}$ (which follows from the assumption that $G$ is discrete).

Before we can state the next preparatory result, we need to establish additional notation. Recall that the boundary of upper half space $\mathbb{H}^{n}$ is identified with $\overline{\mathbb{R}^{n-1}}$. A boundary-cube $Q \subset \mathbb{R}^{n-1}$ is a set of the form $I_{1} \times I_{2} \times \cdots \times I_{n-1}$, where $I_{1}, \ldots, I_{n-1}$ are closed intervals of equal length $c$. We define the extended cube $\widetilde{Q}$ to be the cube 
$Q \times[0, c] \subset \overline{\mathbb{H}^{n}}$ (so $\widetilde{Q}$ is a $n$-cube). Furthermore, for a positive number $M$, the $M$-boundary-cube $M \cdot Q \subset \mathbb{R}^{n-1}$ is the boundary cube of side length $M c$, centered at the same point as $Q$.

The following lemma will allow us to make use of the porosity of the limit set of a discrete QCF group with non-empty regular set in estimating the exponent of convergence of such a group.

Lemma 3.5. Let $G$ be a discrete $K$-quasiconformal group preserving $\mathbb{H}^{n}$ and having non-empty regular set. Assume that $|L(G)| \geq 2$. Then there exists a constant $M>1$ such that the following is true: If $Q \subset \mathbb{R}^{n-1}$ is a boundary-cube whose $M$-boundary-cube $M \cdot Q$ is entirely contained in $\Omega(G)$, then the orbit of $j$ under $G$ does not enter the extended cube $\widetilde{Q}$.

Proof of Lemma 3.5. We will first show that each point in the orbit of $j$ is at a uniformly bounded hyperbolic distance from some hyperbolic geodesic (varying with the orbit point) whose endpoints lie in $L(G)$. Next we will show that if $Q \subset \mathbb{R}^{n-1}$ is a boundary cube whose $M$-boundary-cube $M \cdot Q$ does not intersect $L(G)$, then the hyperbolic distance between the extended cube $\widetilde{Q}$ and any hyperbolic geodesic whose endpoints lie in $L(G)$ is bounded below by some positive constant that only depends on $M$ and the dimension $n$. For large enough $M$ this constant will be so large that the orbit of $j$, which stays close to hyperbolic geodesics with endpoints in $L(G)$, cannot enter the extended cube $\widetilde{Q}$.

For $a, b \in \overline{\mathbb{R}^{n-1}}$ denote by $\beta_{a, b}$ the bi-infinite hyperbolic geodesic in $\mathbb{H}^{n}$ whose Euclidean endpoints are $a, b$. It is a property of quasiconformal mappings preserving $\mathbb{H}^{n}$ that the image $g\left(\beta_{a, b}\right)$ of $\beta_{a, b}$ under a $K$-quasiconformal mapping $g$ preserving $\mathbb{H}^{n}$ lies in a fixed diameter hyperbolic neighborhood of the geodesic $\beta_{g(a), g(b)}$, the size of which only depends on $n$ and $K$ (and not on $a, b$ or $g$ ). This means that there exists a positive constant $m(n, K)$ such that for all $a, b \in \overline{\mathbb{R}^{n-1}}$, all $z \in \beta_{a, b}$, and all $K$-quasiconformal maps $g: \mathbb{H}^{n} \rightarrow \mathbb{H}^{n}$, we have that

$$
\operatorname{dist}^{\text {hyp }}\left(g(z), \beta_{g(a), g(b)}\right) \leq m(n, K) .
$$

We are now ready to show that every point in the orbit of $j$ is at a uniformly bounded hyperbolic distance from some hyperbolic geodesic whose endpoints lie in $L(G)$. Fix two distinct points $a_{0}, b_{0} \in L(G)$, and furthermore fix some point $w_{0} \in \beta_{a_{0}, b_{0}}$. Using the triangle inequality, inequality (3.2), and Theorem 3.3, we obtain that, for any $g \in G$,

$$
\begin{aligned}
\operatorname{dist}^{\text {hyp }}\left(g(j), \beta_{g\left(a_{0}\right), g\left(b_{0}\right)}\right) & \leq \rho\left(g(j), g\left(w_{0}\right)\right)+\operatorname{dist}^{\text {hyp }}\left(g\left(w_{0}\right), \beta_{g\left(a_{0}\right), g\left(b_{0}\right)}\right) \\
& \leq\left(\Phi_{n, K}\left(\rho\left(j, w_{0}\right)\right)+m(n, K)\right)=: C .
\end{aligned}
$$

Note that $\beta_{g\left(a_{0}\right), g\left(b_{0}\right)}$ is a hyperbolic geodesic whose endpoints lie in $L(G)$ since $L(G)$ is invariant under $G$.

Now let $Q \subset \Omega(G)$ be a boundary-cube with center point $\zeta=\left(\zeta_{1}, \ldots, \zeta_{n-1}\right)$, and let $M>1$ such that $M \cdot Q \subset \Omega(G)$ as well. Assume that $Q$ has side length $c$, so that $\widetilde{Q}$ has the form $\left[\zeta_{1}-\frac{c}{2}, \zeta_{1}+\frac{c}{2}\right] \times \ldots \times\left[\zeta_{n-1}+\frac{c}{2}, \zeta_{n-1}+\frac{c}{2}\right] \times[0, c]$. It will be shown, if $M$ is large enough, that for any distinct $a, b \in L(G)$ we have

$$
\operatorname{dist}^{\text {hyp }}\left(\widetilde{Q}, \beta_{a, b}\right) \geq \ln \frac{M}{\sqrt{n+3}} .
$$

Since by (3.3) each orbit point of $j$ has a hyperbolic distance at most $C$ from some hyperbolic geodesic with endpoints in $L(G)$, inequality (3.4) implies that if 
we choose $M$ large enough so that $\ln \frac{M}{\sqrt{n+3}}>C$, then any point in the orbit of $j$ has a positive distance from $\widetilde{Q}$, i.e. the orbit cannot enter $\widetilde{Q}$; thus the proof follows from establishing inequality (3.4).

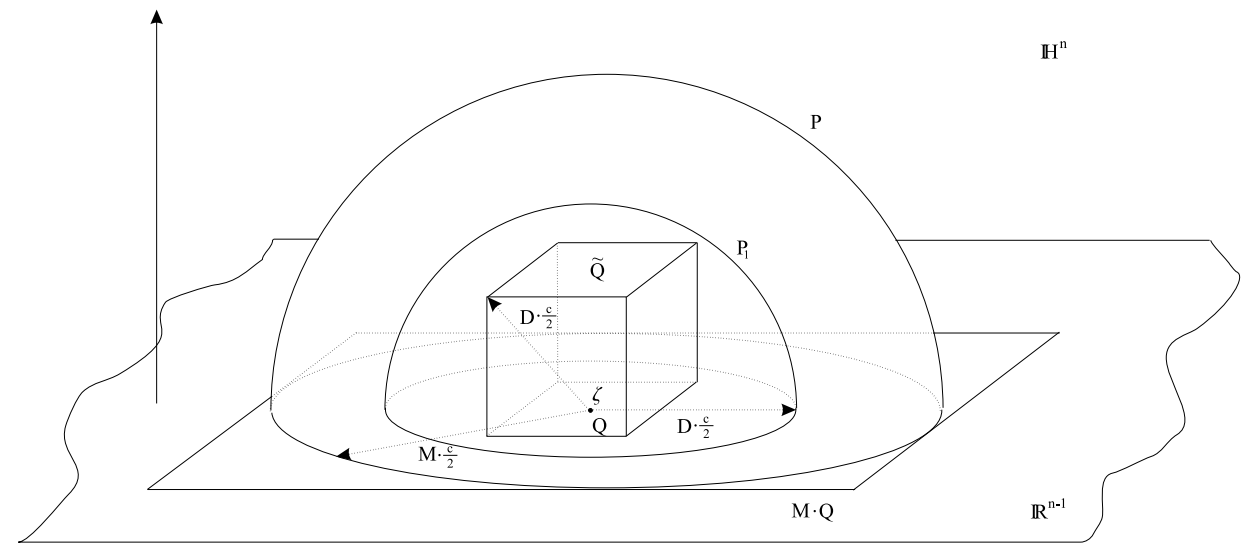

Figure 1

Let $D:=\sqrt{n+3}$. Then the $(n-1)$-hyperbolic plane $P_{1}$ in $\mathbb{H}^{n}$ whose boundary in $\mathbb{R}^{n-1}$ is the Euclidean sphere $\left\{z \in \mathbb{R}^{n-1}:|z-\zeta|=D c / 2\right\}$ circumscribes $\widetilde{Q}$; see Figure 1. Suppose now that $M>D$, and that the $M$-boundary-cube $M \cdot Q$ is entirely contained in the regular set of $G$. Let $P$ be the $(n-1)$-hyperbolic plane in $\mathbb{H}^{n}$ whose boundary in $\mathbb{R}^{n-1}$ is the Euclidean sphere $\left\{z \in \mathbb{R}^{n-1}:|z-\zeta|=M c / 2\right\}$. Then the boundary of $P$ in $\mathbb{R}^{n-1}$ is contained in $M \cdot Q$, and furthermore,

$$
\operatorname{dist}^{\text {hyp }}\left(P_{1}, P\right)=\ln \frac{M}{D} \text {. }
$$

Since $P_{1}$ circumscribes $\widetilde{Q}$ we obtain from this that

$$
\operatorname{dist}^{\text {hyp }}(\widetilde{Q}, P) \geq \ln \frac{M}{D} .
$$

Because $\left\{z \in \mathbb{R}^{n-1}|| z-\zeta \mid \leq M c / 2\right\}$ is contained in $M \cdot Q$ which by itself is contained in the regular set of $G$, we have that for any two distinct points $a, b \in L(G)$ the hyperbolic geodesic $\beta_{a, b}$ lies in the hyperbolic half-space whose boundary is $P$, and that does not contain $\widetilde{Q}$. Hence

$$
\operatorname{dist}^{\operatorname{hyp}}\left(\widetilde{Q}, \beta_{a, b}\right) \geq \ln \frac{M}{D}
$$

as desired.

As our final preparation for the proof of Theorem 1.2, we detail a counting result to aid us in the calculation of the exponent of convergence. Recall that $G$ is taken to be a discrete QCF group acting on $\overline{\mathbb{R}^{n}}$ and preserving $\mathbb{H}^{n}$. In [7] we developed a localized version of the exponent of convergence by defining, for each $x \in \overline{\mathbb{R}^{n-1}}=\partial \mathbb{H}^{n}$,

$$
\delta_{x}(G):=\lim _{r \rightarrow 0} \inf \left\{s>0 \mid \sum_{g \in G: g(j) \in D^{\text {chord }}(x, r)} e^{-s \rho(j, g(j))}<\infty\right\},
$$


where $j=(0,0, \ldots, 0,1) \in \mathbb{H}^{n}$ and $D^{\text {chord }}(x, r)$ is the chordal $n$-dimensional ball of chordal radius $r$ around $x \in \overline{\mathbb{R}^{n-1}} \subset \overline{\mathbb{R}^{n}}$. This $\delta_{x}(G)$ is called the local exponent of convergence. The value of $\delta_{x}(G)$ does not depend on the choice of the orbit point $j$, and we further show in [7] that $\delta_{x}(G)=0$ for $x \notin L(G)$ and $\delta(G)=$ $\max _{x \in L(G)} \delta_{x}(G)$; in particular the local exponent need not be constant on $L(G)$ for $K$-quasiconformal groups $G$ with $K>1$. See [7] for a comprehensive discussion of the local exponent of convergence. (For a Kleinian group $\Gamma$ it is true that $\delta(\Gamma)=\delta_{x}(\Gamma)$ for all $x \in L(\Gamma)$.)

The exponent of convergence can be calculated by asymptotically counting the number of points of the orbit of the point $j$ that lie inside a hyperbolic ball of radius $R$, centered at $j$. Let $N(R):=\#(G(j) \cap B(j, R))$, where $B(j, R)$ is the open ball of hyperbolic radius $R$ about $j$. Then (see Proposition 2.2 in [6])

$$
\delta(G)=\limsup _{R \rightarrow \infty} \frac{\log N(R)}{R} .
$$

We will now show how to use (3.5) to localize the analysis around a point $x_{0} \in L(G)$. By conjugating with a Möbius transformation we may assume that $x_{0}=0 \in \overline{\mathbb{R}^{n-1}}$.

Let $I:=[-0.5,0.5] \times \cdots \times[-0.5,0.5] \subset \mathbb{R}^{n-1}$ be the unit cube in $\mathbb{R}^{n-1}$ centered at 0 , and define the layers $A_{k} \subset \mathbb{H}^{n}, k \in \mathbb{N}$, by

$$
A_{k}:=\left\{(x, t) \in \mathbb{H}^{n} \mid x \in I \text { and } 2^{-k-1} \leq t<2^{-k}\right\} .
$$

Then points in the layer $A_{k}$ have an approximate hyperbolic distance $k \log 2$ from $j$. Explicitly, via the triangle inequality, it is immediate that each point in $A_{k}$ is at least distance $k \log 2$ from $j$, and is at most the distance $k \log 2$ plus half the diameter of the closed set $\left\{\left(x_{1}, \ldots, x_{n-1}, 1\right) \in \mathbb{H}^{n}: \frac{-1}{2} \leq x_{i} \leq \frac{1}{2}\right.$ for $\left.1 \leq i \leq n-1\right\}$; note in particular that this additive constant applied to $k \log 2$ is independent of the index $k$. Let $\# A_{k}:=\#\left(G(j) \cap A_{k}\right)$. The next proposition follows directly from (3.5).

Proposition 3.6. Let $G$ be a discrete $Q C F$ group acting on $\overline{\mathbb{R}^{n}}$ and fix $x_{0} \in L(G) \subset$ $\overline{\mathbb{R}^{n-1}}$. Normalizing so that $x_{0}=0$, and defining $I, A_{k}$, and $\# A_{k}$ as done directly above, we have that

$$
\delta_{0}(G) \leq \limsup _{k \rightarrow \infty} \frac{\log \left(\# A_{k}\right)}{k \log 2} \leq \delta(G) .
$$

We are finally ready to prove Theorem 1.2. Recall that $G$ is assumed to be a discrete quasiconformal group that preserves $\mathbb{H}^{n}$, that has a non-empty regular set, and that has a purely conical limit set. Our goal is to show that $\delta(G)<n-1$.

First we provide an overview of the strategy used in establishing the theorem. The argument is by contradiction. Using the assumption that $\Omega(G) \neq \emptyset$, we will show that there is too little hyperbolic space in the layers $A_{k}$ above a point $x_{0}$ where the local exponent is maximized, in order for this local exponent to be equal to $n-1$. The key geometric idea is to use the fact that $L(G)$ is porous (see Corollary 3.2). In particular, we obtain the existence of an integer $q$, such that if we divide any cube in $\mathbb{R}^{n-1}$ into $q^{n-1}$ sub-cubes of equal side length then at least one of these subcubes is entirely contained in the regular set. Inductively, by further subdivision, we construct a collection of cubes that do not intersect $L(G)$. By extending these cubes to cubes in $\mathbb{H}^{n}$, and possibly increasing $q$, we will see that the orbit of $j$ cannot enter these extended cubes. But this means that the part of the orbit of $j$ 
that we are interested in is restricted to being in $A_{k}$ minus the space taken away by the extended cubes. We will calculate that the hyperbolic space available in $A_{k}$ for orbit points is thus reduced by a factor $\lambda^{k}$, where $\lambda<1$. From this it will follow that (3.6) cannot be satisfied with $\delta_{0}(G)=\delta(G)=n-1$. We will now make this description of the proof precise.

Proof of Theorem 1.2. The proof of the theorem is by contradiction, and hence we assume that $\delta(G)=n-1$. Thus we can find a point $x_{0} \in L(G)$ such that $\delta_{x_{0}}(G)=n-1$, and by conjugating $G$ with a Möbius transformation (which does not change the exponent of convergence) we may assume that $x_{0}=0 \in \overline{\mathbb{R}^{n-1}}=\partial \mathbb{H}^{n}$, and furthermore that $\infty \in \Omega(G)$. Defining $I, A_{k}$ and $\# A_{k}$ as above, we then obtain from Proposition 3.6 that

$$
\limsup _{k \rightarrow \infty} \frac{\log \left(\# A_{k}\right)}{k \log 2}=n-1 .
$$

In order to fill in the details as given in our overview, we first note that the hyperbolic volume of $A_{k}$ is

$$
\operatorname{vol}^{\text {hyp }}\left(A_{k}\right)=\frac{2^{n-1}-1}{n-1}\left(2^{n-1}\right)^{k} .
$$

¿From the hyperbolic uniform separation of the orbit of $j$ (see Proposition 3.4) we obtain that the maximum number of orbit points that could fit into $A_{k}$ is bounded above by the volume of $A_{k}$ times a constant depending only on $n$ and the hyperbolic separation, but independent of $k$. That is, (3.8) gives

$$
\# A_{k} \leq C\left(2^{n-1}\right)^{k}
$$

where $C$ depends on the dimension $n$ and the hyperbolic separation of the orbit of $j$, but not on $k$.

Now we will demonstrate that the porosity of the limit set of $G$ restricts the space in $A_{k}$ which is available for the orbit of $j$. Recall that since $L(G)$ is porous (see Corollary 3.2), there exists an integer $q$ such that if we divide any cube in $\mathbb{R}^{n-1}$ into $q^{n-1}$ sub-cubes of equal side length then at least one of these sub-cubes is entirely contained in $\Omega(G)$. Dividing the unit cube $I$ defined above into $q^{n-1}$ sub-cubes of equal side length we can find (at least) one cube of side length $q^{-1}$ that does not intersect $L(G)$. If we divide each of the remaining $\left(q^{n-1}-1\right)$ sub-cubes into $q^{n-1}$ sub-sub-cubes then in each sub-cube we can find at least one sub-sub-cube that does not intersect $L(G)$, i.e. altogether we can find, in addition to the cube found above, $\left(q^{n-1}-1\right)$ cubes of side length $q^{-2}$ that do not intersect $L(G)$. Continuing inductively in this manner we can find

$$
\left(q^{n-1}-1\right)^{i-1} \text { cubes of side length } q^{-i}, i=1,2,3, \ldots,
$$

and all these cubes $Q$ have disjoint interiors and are entirely contained in $\Omega(G)$. Using Lemma 3.5 and enlarging the integer $q$ if necessary (so that all $M$-cubes $M Q$ are still disjoint from $L(G)$, where $M$ is the value from Lemma 3.5) we furthermore obtain that the extended cubes $\widetilde{Q} \subset \overline{\mathbb{H}^{n}}$ do not contain any points of the orbit of $j$.

However, this affects the maximum number of orbit points that can lie in the sets $A_{k}$. To be precise, there exists a fixed integer $N$, depending only on $q$, such that for each $m \in \mathbb{N}$, the space in $A_{m N}$ gets reduced at least by all $\left(q^{n-1}-1\right)^{i-1}$ 
extended cubes of side length $q^{-i}$ for all $i=1, \ldots, m$. The total volume of the intersection of the boundaries of all these extended cubes with $\mathbb{R}^{n-1}$ is at least

$$
V_{m}=\sum_{i=1}^{m}\left(q^{n-1}-1\right)^{i-1} \cdot\left(q^{-i}\right)^{n-1}=1-\left(\frac{q^{n-1}-1}{q^{n-1}}\right)^{m} .
$$

Thus the volume of $I$ minus these cubes is $\left(\frac{q^{n-1}-1}{q^{n-1}}\right)^{m}$, and so the hyperbolic volume that is available inside $A_{m N}$ for the orbit of $j$ is only $\lambda^{m} \cdot \operatorname{vol}^{\text {hyp }}\left(A_{m N}\right)$, where $\lambda=\left(\frac{q^{n-1}-1}{q^{n-1}}\right)^{m}<1$. Using (3.9) this implies that the number of orbit points that fit into $A_{m N}$ is in fact bounded above by

$$
\# A_{m N} \leq C \lambda^{m}\left(2^{n-1}\right)^{m N} .
$$

Similarly, one obtains the bounds

$$
\# A_{m N+i} \leq C \lambda^{m}\left(2^{n-1}\right)^{m N+i} \text { for } i=0, \ldots, N-1 \text { and each } m \in \mathbb{N} .
$$

But using (3.10) we now finally obtain a contradiction to (3.7), since

$$
\begin{aligned}
\frac{\log \left(\# A_{m N+i}\right)}{(m N+i) \log 2} & \leq \frac{\log \left(C \lambda^{m}\left(2^{n-1}\right)^{m N+i}\right)}{(m N+i) \log 2} \\
& =\frac{\log C+m \log \lambda+(n-1)(m N+i) \log 2}{(m N+i) \log 2} \\
& \stackrel{m \rightarrow \infty}{\longrightarrow} \frac{\log \lambda}{N \log 2}+n-1<n-1 .
\end{aligned}
$$

Since this is true for each $i=0, \ldots, N-1$ we obtain the desired contradiction to (3.7).

As with the Hausdorff dimension result, Theorem 1.3 does not generalize to the full convergence group setting. Indeed, there is an example (see Example 5.1 in [6]) of a purely conical convergence group preserving $\mathbb{B}^{n}$, having non-empty regular set, and so that the exponent of convergence (calculated from the center of the ball) of the group is infinite.

Tukia extends to convergence groups ([26]), in the presence of parabolic elements, various of Bowditch's equivalent definitions([9]) of geometric finiteness for Kleinian groups. We conjecture that both Theorem 1.3 and Theorem 1.2 are true for cusp uniform discrete quasiconformal groups:

Conjecture 3.7. Let $G$ be a discrete quasiconformal group preserving $\mathbb{H}^{n}$ with nonempty regular set. Assume that $L(G)$ consists only of conical limit points and bounded parabolic points (i.e. $G$ is cusp-uniform in the sense of Bowditch/Tukia).

Then $\delta(G)<n-1$ and $\operatorname{dim} L(G)<n-1$.

\section{Infinite-Index Subgroups}

A surprising amount of information persists when the conditions that a group action be discrete and isometric are relaxed to the condition that the group action be merely discrete, see e.g. [15], [4], and [5]. In this spirit, and as an application of our results, we will consider how infinite-index subgroups reside within discrete quasiconformal groups. Recall the following: 
Theorem 4.1. (Canary-Taylor [12]) Let $\Gamma$ be a non-elementary Kleinian group, and suppose that $\hat{\Gamma}$ is an infinite-index, geometrically finite subgroup of $\Gamma$. Then

$$
\delta(\hat{\Gamma})<\delta(\Gamma) \quad \text { and } \quad \operatorname{dim} L(\hat{\Gamma})<\operatorname{dim} L(\Gamma) .
$$

We give a brief synopsis of the proof of this fact. First a theorem of Susskind and Swarup ([22]) is used to assert that the limit set of $\hat{\Gamma}$ is properly contained in the limit set of $\Gamma$. Thus we can find a (necessarily) geometrically finite Kleinian group $\Gamma^{\prime}$ that is the Klein combination of $\hat{\Gamma}$ with a loxodromic element cyclic group $\langle\gamma>\subset \Gamma \backslash \hat{\Gamma}$. Now we can use a theorem of Furasawa ([13]) asserting, under these conditions, that $\delta(\hat{\Gamma})<\delta\left(\Gamma^{\prime}\right)$. The result now follows immediately from Patterson-Sullivan theory.

While we are able to generate analogues for both the Susskind-Swarup theorem and the Klein combination theorem for discrete quasiconformal groups, the last two steps do not generalize. Indeed, we are only able to establish a relative version of Theorem 4.1; an example to be given below ([2]) shows that this theorem in the quasiconformal setting is not true in its full generality.

We first show:

Theorem 4.2. Let $G$ be a non-elementary discrete convergence group acting on a compact metric space. Let $H$ be a subgroup of $G$ with purely conical limit set. If $L(G)=L(H)$, then the index of $H$ in $G$ is finite.

Proof of Theorem 4.2. We first observe that since $L(H)$ is purely conical, so is $L(G)$, since both these sets are equal and $H \subset G$. Let $T_{G}$ denote the triple space over $M=L(G)$, i.e. $T_{G}=T(L(G))=\left\{\left(x_{1}, x_{2}, x_{3}\right) \mid x_{1}, x_{2}, x_{3} \in L(G)\right.$ distinct $\}$. Then the discrete action of $G$ on the compact, perfect metric space $L(G)$ extends to a discontinuous action on $T_{G}$, and by Theorem $2.1, T_{G} / G$ is compact. Similarly we define $T_{H}$ to be the triple space over $L(H)$ and obtain that $T_{H} / H$ is compact. We note that $T_{G}=T_{H}$ by assumption; let $T:=T_{G}$. Since $G$ and $H$ act properly discontinuously on $T$, we have that $T / H$ is a cover of $T / G$, and since both these spaces are compact, the cover must be finite. This implies that the index of $H$ in $G$ is finite.

Remark 4.3. See Susskind and Swarup [22]; it seems likely that this result can be generalized to allow $H$ to be a cusp-uniform discrete convergence group.

We now have the following analogous versions of Theorem 4.1. By combining Theorem 1.3 with Theorem 4.2, we have:

Theorem 4.4. Let $G$ be a discrete quasiconformal group acting on $\overline{\mathbb{R}^{n}}$ with $L(G)=$ $\overline{\mathbb{R}^{n}}$. If $H$ is an infinite-index subgroup of $G$ with purely conical limit set then $\operatorname{dim} L(H)<\operatorname{dim} L(G)=n$.

We say that a QCF group $G$ acting on $\mathbb{H}^{n}$ is of divergence type if its Poincaré series diverges at $n-1$. It is a result of Garnett-Gehring-Jones [14] that such groups have the property that their conical limit set is of full measure in $\overline{\mathbb{R}^{n-1}}$. Thus, by Theorem 2.7 in [6] and a lemma in [16] (see also Lemma 2.3 in [6]), we have that $\delta(G)=n-1$. By combining Theorem 1.2 with Theorem 4.2, we can thus assert:

Theorem 4.5. Let $G$ be a discrete QCF group of divergence type. If $H$ is an infinite-index subgroup of $G$ with purely conical limit set then $\delta(H)<\delta(G)$. 
One might hope with a different set of arguments that the condition $L(G)=$ $\overline{\mathbb{R}^{n}}$ could be removed from Theorem 4.4, thus giving (at least for infinite-index subgroups with purely conical limit sets) a full analogue to Theorem 4.1. However, the following example, due to Chris Bishop [2], shows that such an analogue is not possible.

Example 4.6. Infinite-index geometrically finite subgroups of discrete quasiconformal groups do not, in general, have limit sets of Hausdorff dimension strictly less than the Hausdorff dimension of the full group.

Construction. Suppose $\Gamma$ is a Kleinian Fuchsian group acting on $\overline{\mathbb{R}^{2}}$ with limit set the whole circle, and $\widetilde{\Gamma}$ is a finitely generated non-elementary subgroup whose limit $L(\widetilde{\Gamma})$ set is a Cantor set. Then a standard result say that $\operatorname{dim} L(\widetilde{\Gamma})>0$ (e.g. see [20]). Furthermore, the dimension of $L(\widetilde{\Gamma})$ can be raised by a conjugation with a quasiconformal mapping $\varphi$ of the plane so that $\varphi(L(\widetilde{\Gamma}))$ has any dimension strictly between $\operatorname{dim}(\widetilde{\Gamma})$ and 2 , see [1]. Moreover, this map $\varphi$ may be chosen to be smooth off $L(\widetilde{\Gamma})$ and so that $\operatorname{dim} L(\varphi(\Gamma))=\operatorname{dim} L(\varphi($ circle $))=\operatorname{dim}(\varphi(L(\widetilde{\Gamma})))$. Thus conjugating $\Gamma$ by this map gives a discrete quasiconformal group $G=\varphi \Gamma \varphi^{-1}$ with an infinite-index non-elementary geometrically finite subgroup $\widetilde{G}=\varphi \widetilde{\Gamma} \varphi^{-1}$ so the limit sets of both groups have the same Hausdorff dimension.

\section{Remark 4.7.}

1) As in Conjecture 3.7, we expect that the above results are true under the presence in $G$ of bounded parabolic elements.

2) The assumption that $G$ be a discrete quasiconformal convergence group in Theorem 4.4 is probably necessary. There is an example ([6]) of a discrete convergence group $G$ acting on $\overline{\mathbb{R}^{2}}$ having an infinite-index subgroup $\tilde{G}$, such that $\operatorname{dim} L(\tilde{G})=\operatorname{dim} L(G)$. However, in this example the group $G$ has a non-empty regular set. It can be shown that the groups are not quasiconformal.

We finish this note with the following positive example, and a resulting question.

Example 4.8. There exists a (non-Kleinian) quasiconformal group $G$ acting on $\overline{\mathbb{R}^{2}}$ that has an infinite-index non-elementary geometrically finite subgroup $\widetilde{G}$ so that $\operatorname{dim} L(\widetilde{G})<\operatorname{dim} L(G)$, and $\delta(\widetilde{G})<\delta(G)$.

Construction. Let $\widetilde{\Gamma}$ be a non-elementary geometrically finite Fuchsian group acting on the plane (i.e. $L(\widetilde{\Gamma}) \subset \partial \mathbb{D})$. Let $\gamma$ be a "handle" to be added to $\widetilde{\Gamma}$ via the Klein Combination Theorem [18], i.e. $\gamma$ is a Möbius transformation so that $\Gamma:=\langle\widetilde{\Gamma}, \gamma\rangle=\widetilde{\Gamma} \star\langle\gamma\rangle$ is a free product. We suppose, furthermore, that the isometric circles of $\gamma$ and $\gamma^{-1}$ are disjoint and are contained in a fundamental domain in a component of the regular set of $\widetilde{\Gamma}$. Let $D$ be the finite 2-component Euclidean disk bounded by the isometric circles of $\gamma$. Then $D$ is moved off of itself under all elements of $\widetilde{\Gamma}$. For each $n \in \mathbb{N}$, define a Beltrami coefficient $\mu_{n}: \mathbb{C} \rightarrow \mathbb{C}$ by

$$
\mu_{n}(z):= \begin{cases}\frac{1}{n} & \text { if } z \in D, \\ \frac{g^{\prime}(z)}{g^{\prime}(z)} \mu_{n}(g(z)) & \text { if } g(z) \in D \text { for some } g \in \widetilde{\Gamma} \\ 0 & \text { otherwise. }\end{cases}
$$


Let $\varphi_{n}: \mathbb{C} \rightarrow \mathbb{C}$ be the normalized solution to the Beltrami equation, i.e. $\varphi_{n}$ is the quasiconformal mapping that satisfies the Beltrami equation $\left(\varphi_{n}\right)_{\bar{z}}=\mu\left(\varphi_{n}\right)_{z}$ and fixes $0,1, \infty$. Then by construction, $\varphi_{n}$ is compatible with $\widetilde{\Gamma}$, but not with $\gamma$, hence $\widetilde{G}_{n}:=\varphi_{n} \widetilde{\Gamma} \varphi_{n}^{-1}$ is a Möbius group, but $\varphi_{n} \gamma \varphi_{n}^{-1}$ is not a Möbius transformation. Thus

$$
G_{n}:=\varphi_{n} \Gamma \varphi_{n}^{-1}=\widetilde{G}_{n} \star\left\langle\varphi_{n} \gamma \varphi_{n}^{-1}\right\rangle
$$

is a non-Kleinian discrete quasiconformal group that has an infinite-index nonelementary geometrically finite subgroup $\widetilde{G}_{n}$ which is Möbius.

By construction we know that the quasiconformal dilatation of $\mu_{n}$ converges to 1 as $n \rightarrow \infty$. From a result in Kleinian groups ([12], Theorem 1) we know that

$$
\delta(\widetilde{\Gamma})<\delta(\Gamma) \quad \text { and equivalently } \operatorname{dim} L(\widetilde{\Gamma})<\operatorname{dim} L(\Gamma) .
$$

Using a theorem on the distortion of the exponent of convergence under quasiconformal conjugation (see Theorem 1.2 in [8]) we conclude that

$$
\delta\left(\widetilde{G}_{n}\right)<\delta\left(G_{n}\right)
$$

for large enough $n$. Using the corresponding distortion bounds for the change in the Hausdorff dimension under a quasiconformal mapping (see [17]), we also obtain that

for large enough $n$.

$$
\operatorname{dim} L\left(\widetilde{G}_{n}\right)<\operatorname{dim} L\left(G_{n}\right)
$$

Given the contrasting natures of Bishop's example (Example 4.6) and Example 4.8, we are motivated to ask:

Question 4.9. Let $G$ be a discrete QCF group that preserves $\mathbb{H}^{n}$, and that has non-empty regular set in $\overline{\mathbb{R}^{n-1}}$. Suppose that $H$ is an infinite index cusp uniform subgroup of $G$.

What are the necessary and sufficient conditions, so that $\delta(H)<\delta(G)$ and/or $\operatorname{dim} L(H)<\operatorname{dim} L(G)$ ?

\section{REFERENCES}

1. C.J. Bishop, Quasiconformal maps which increase dimension, Ann. Acad. Sci. Fenn. 24 (1999) 397-407.

2. C.J. Bishop, Personal communication.

3. C.J. Bishop and P. Jones, Hausdorff dimension and Kleinian groups, Acta Math. 179 (1997) 1-39.

4. P. Bonfert-Taylor, Jørgensen's inequality for discrete convergence groups, Ann. Sci. Fenn. 25 (2000) 131-150.

5. P. Bonfert-Taylor and G. Martin, Quasiconformal groups with small dilatation I, Proc. Amer. Math. Soc. 129 (2000) 2019-2029.

6. P. Bonfert-Taylor and E.C. Taylor, Hausdorff dimension and limit sets of quasiconformal groups, Mich. Math. J. 49 (2001) 243-257.

7. P. Bonfert-Taylor and E.C. Taylor, Patterson-Sullivan theory and local analysis of limit sets, to appear in Trans. Amer. Math. Soc.

8. P. Bonfert-Taylor and E.C. Taylor, The exponent of convergence and a theorem of Astala, Indiana Univ. Math. J. 51(3) (2002), 607-623.

9. B. Bowditch, Geometrical finiteness for hyperbolic groups, J. Funct. Anal. 113 (1993) 245-317.

10. B. Bowditch, A topological characterization of hyperbolic groups, J. Amer. Math. Soc. 11 (1998) 643-667.

11. R.D. Canary, Y.N. Minsky, E.C. Taylor, Spectral theory, Hausdorff dimension and the topology of hyperbolic 3-manifolds, J. Geom. Anal. 9 (1999) 18-40. 
12. R.D. Canary and E.C. Taylor, Kleinian groups with small limit sets, Duke Math. J. 73 (1994) 371-381.

13. H. Furasawa, The exponent of convergence of Poncaré series of combination groups, Tôhoku Math. J. (2) 43 (1991) 1-7.

14. J.B. Garnett, F.W. Gehring, and P.W. Jones, Quasiconformal groups and the conical limit set, Holomorphic functions and moduli, Vol. II, 59-67, Math. Sci. Res. Inst. Publ. 11, Springer-Verlag 1988.

15. F.W. Gehring and G.J. Martin, Discrete quasiconformal groups I, Proc. London Math. Soc. (3) $\mathbf{5 5}$ (1987) 331-358.

16. F.W. Gehring and G.J. Martin, Discrete quasiconformal groups II, unpublished manuscript.

17. F.W. Gehring and J. Väisälä, Hausdorff dimension and quasiconformal mappings, J. London Math. Soc. (2) 6 (1973) 504-512.

18. B. Maskit, Kleinian groups, Springer-Verlag, 1987.

19. P. Mattila, Geometry of sets and measures in Euclidean spaces. Fractals and rectifiability. Cambridge Studies in Advanced Mathematics, 44. Cambridge University Press, Cambridge, 1995.

20. P.J. Nicholls, The Ergodic Theory of Discrete Groups, Cambridge University Press, 1989.

21. D. Sullivan, On the ergodic theory at infinity of an arbitrary discrete group of hyperbolic motions, Riemann surfaces and related topics: proceedings of the 1978 Stony Brook Conference, Annals of Mathematics Studies 97, Princeton University Press, 1981, 465496.

22. P. Susskind and G. Swarup, Limit sets of geometrically finite hyperbolic groups, Amer. J. Math. 114 (1992) 233-250.

23. D. Sullivan, Entropy, Hausdorff measures old and new, and limit sets of geometrically finite Kleinian groups, Acta Math. 153 (1984) 259-277.

24. P. Tukia, A quasiconformal group not isomorphic to a Möbius group, Ann. Acad. Sci. Fenn. Ser. AI Math. 10 (1985) 561-562.

25. P. Tukia, The Hausdorff dimension of the limit set of a geometrically finite Kleinian group, Acta Math. 152 (1984) 127-140.

26. P. Tukia, Conical limit points and uniform convergence groups J. Reine Angew. Math. 501 (1998) 71-98.

27. J. Väisäilä, Lectures on n-Dimensional Quasiconformal Mappings, Springer-Verlag 1971.

Faculty of Mathematical Studies, University of Southampton, Southampton, EngLAND

E-mail address: j.w.anderson@maths.soton.ac.uk

Wesleyan University, Department of Mathematics and Computer Science, MiddleTOWN, CT

E-mail address: pbonfert@wesleyan.edu

Wesleyan University, Department of Mathematics and Computer Science, MiddleTOWN, CT

E-mail address: ectaylor@wesleyan.edu 